- (c) 2004 American Chemical Society, Org. Lett., Gao ol040043a Supporting Info Page 1

\title{
Efficient Preparation of Glycoclusters from Silsesquioxanes
}

Yongjun $\mathrm{Gao}^{\ddagger}$, Atsuko Eguchi ${ }^{\dagger}$, Kazuaki Kakehi ${ }^{\dagger}$, and Yuan C. Lee ${ }^{\star \ddagger}$

Department of Biology, Johns Hopkins University, 3400 North Charles

Street, Baltimore, MD 21218, USA, and Faculty of Pharmceutical Sciences,

Kinki University, Kowakae 3-4-1, Higashi-Osaka 577-8502, Japan

yclee@jhu.edu

\section{Supporting Information}

\section{General Procedures}

2. Synthesis and Spectroscopic Data

3. Lectin Binding Assay

4. Reference

5. ${ }^{1} \mathrm{H}$ NMR, ${ }^{13} \mathrm{C}$ NMR and MALDI-TOF MS spectra 
General Procedures Chemicals were from Aldrich (Milwaukee, WI) and used as received. All photoadditions were performed in borosilicate glass flasks and irradiated at $254 \mathrm{~nm}$ with a UV Lamp (115 V, 0.16 Amps, bulb 10x110 mm). Column chromatography was carried out using E. Merck Silica Gel 60 F (230-400 mesh). Analytical thin-layer chromatography (TLC) was performed on aluminum sheets coated with silica gel $60 \mathrm{~F}_{254}(0.25 \mathrm{~mm}$ thickness, E. Merck). Detection of the compounds was by quenching of fluorescence and/or charring with $15 \% \mathrm{H}_{2} \mathrm{SO}_{4}$ in ethanol solution followed by heating at $180^{\circ} \mathrm{C}$. The ratios of solvents used for TLC are expressed in vol/vol.

Gel filtration chromatography was performed using either (1) a column packed with Sephadex G-25 $(5 \times 200 \mathrm{~cm})$ eluting with $0.1 \mathrm{M}$ acetic acid or (2) a column packed with Sephadex G-15 $(2 \times 145 \mathrm{~cm})$ eluting with distilled water. Melting points were determined with a Fisher-Johns apparatus and are not corrected. ${ }^{1} \mathrm{H}$ NMR and ${ }^{13} \mathrm{C}$ NMR spectra were recorded with a Varian-400 NMR spectrometer at nominal resonance frequencies of 400 $\mathrm{MHz}$ and $100 \mathrm{MHz}$ respectively in $\mathrm{CDCl}_{3}$ (referenced to internal $\mathrm{Me}_{4} \mathrm{Si}$ at $\delta_{\mathrm{H}} 0 \mathrm{ppm}, \delta_{\mathrm{c}} 0$ ppm) and $\mathrm{D}_{2} \mathrm{O}$ (referenced to internal acetone at $\delta_{\mathrm{H}} 2.225 \mathrm{ppm}, \delta_{\mathrm{c}} 29.8 \mathrm{ppm}$ ). The chemical shifts $(\delta)$ were in parts per million (ppm). First order $J$ values were given in $\mathrm{Hz}$. Fast atom bombardment mass spectra (FAB MS) data were obtained using $m$-nitrobenzyl alcohol as matrix. Matrix-assisted laser desorption ionization time-of-flight mass spectra (MALDI-TOF-MS) were recorded with a MALDI-Compact (Kratos) instrument in the positive mode using 2, 5-dihydroxybenzoic acid (DHB) as matrix unless otherwise indicated and an average of 50 laser shots per sample.

$N$-Benzyloxycarbonyl 5-aminopetanol $2^{1}$ and $N$-fluorenylmethoxycarbonyl-6aminohexanal $9^{2}$ were prepared according to the known methods. 


\section{5-(Benzyloxycarbonylamino)pentyl 2,3,4, 6-tetra-acetyl-a-D-mannopyranoside $(3)^{3}$}

This compound was prepared by a method similar to that described in ref 3 .

Tin(IV) chloride $(0.75 \mathrm{ml}, 6.4 \mathrm{mmol})$ was added dropwise over $20 \mathrm{~min}$ to a solution of 1,2,3,4, 6-penta-acetyl- $\alpha$-D-mannopyranoside (2.28 g, $5.95 \mathrm{mmol})$ and 5-

(benzyloxycarbonylamino)petanol $2^{1}(1.0 \mathrm{~g}, 3.98 \mathrm{mmol})$ in dichloromethane $(50 \mathrm{ml})$, and the mixture was stirred for $12 \mathrm{~h}$. It was then poured into ice-cold aqueous sodium bicarbonate $(50 \mathrm{ml})$ with vigorous stirring, and the resulting precipitate was removed by filtration through a pad of on a Celite. The filtrate was extracted with choloroform, and the combined extracts, were dried and concentrated. The residual syrup was purified on silica gel (5:4 Hexanes/EtOAc as eluent) to give 3 as a colorless syrup ( $1.82 \mathrm{~g}, 55 \%)$.

${ }^{1} \mathrm{H}$ NMR $\left(400 \mathrm{MHz}, \mathrm{CDCl}_{3}\right.$, r. t.) $\delta 5.35(\mathrm{dd}, 1 \mathrm{H}, \mathrm{J}=3.6$ and $10.0 \mathrm{~Hz}, \mathrm{H}-3), 5.27((\mathrm{t}, 1$ $\mathrm{H}, J=10.0 \mathrm{~Hz}, \mathrm{H}-4), 5.23$ (br. d, $1 \mathrm{H}, J=3.6 \mathrm{~Hz}, \mathrm{H}-2), 5.10$ (s, $2 \mathrm{H}, \mathrm{PhCH}_{2}$ ), 4.79 (d, $1 \mathrm{H}$, $\mathrm{J}=1.6 \mathrm{~Hz}, \mathrm{H}-1), 4.28(\mathrm{dd}, 1 \mathrm{H}, \mathrm{J}=5.3 \mathrm{~Hz}$ and $12.0 \mathrm{~Hz}, \mathrm{H}-6 \mathrm{~b}), 4.10(\mathrm{dd}, 1 \mathrm{H}, \mathrm{J}=2.4 \mathrm{~Hz}$ and $12.0 \mathrm{~Hz}, \mathrm{H}-6 \mathrm{a}), 3.98$ (ddd, $1 \mathrm{H}, \mathrm{J}=2.4,5.3$, and $10.0 \mathrm{~Hz}, \mathrm{H}-5), 3.70-3.67\left(\mathrm{~m}, 1 \mathrm{H}, \mathrm{OC} H_{2}\right)$, 3.46-3.44 (m, 1H, $\left.\mathrm{OCH}_{2}\right), 3.24-3.19\left(\mathrm{~m}, 2 \mathrm{H}, \mathrm{CH}_{2} \mathrm{NHCbz}\right), 2.16,2.09,2.05,1.99(4 \mathrm{~s}, 12$ $\left.\mathrm{H}, \mathrm{CH}_{3} \mathrm{CO}\right), 1.63(\mathrm{~m}, 2 \mathrm{H}), 1.55(\mathrm{~m}, 2 \mathrm{H}), 1.40(\mathrm{~m}, .2 \mathrm{H})$.

${ }^{13} \mathrm{C} \mathrm{NMR}\left(100 \mathrm{MHz}, \mathrm{CDCl}_{3}\right.$, r. t.): $\delta 170.70,170.13,169.96,169.79$ (4 CO), 156.43 $\left(\mathrm{PhCH}_{2} \mathrm{OCO}\right), 136.65,128.52,128.09,97.55,69.69,69.11,68.44,68.27,66.62,66.28$, $62.57,60.39,40.92,29.77,28.87,23.40,21.05,20.93,20.77,20.72$.

\section{5-Aminopentyl $\alpha$-D-mannopyranoside (4)}

The fully protected lactoside $3(3.75 \mathrm{~g}, 6.6 \mathrm{mmol})$ was dissolved in dry methanol (50 $\mathrm{ml}$ ), and the solution was made basic ( $\mathrm{pH} 11$ by moist $\mathrm{pH}$ paper) by addition of solid $\mathrm{NaOMe}$ (about $50 \mathrm{mg}$ ), and the mixture was left at room temperature. When the reaction 
was complete $(6 \mathrm{~h})$, as shown by TLC, $\mathrm{H}_{2} \mathrm{O}(20 \mathrm{ml})$ was added to dissolve the precipitates formed, and the reaction was stirred for a further $2 \mathrm{~h}$, during which time the precipitate dissolved completely. The reaction mixture was neutralized with Dowex50WX8-400 $\left(\mathrm{H}^{+}\right.$form $)$resin and filtered. The filtrate and washings were concentrated to ca. $15 \mathrm{ml}$, and then acetic acid $(10 \mathrm{ml})$ and $\mathrm{Pd} / \mathrm{C} 10 \%(600 \mathrm{mg})$ were added, whereafter the mixture was hydrogenated at atmospheric pressure for $12 \mathrm{~h}$. After completion of (TLC chloroform/methanol, 1:1), the mixture was filtered through a pad of Celite, washed with water $(30 \mathrm{ml})$, concentrated to $\sim 3 \mathrm{ml}$, and fractionated on a Sephadex G-25 column ( $5 \times 200 \mathrm{~cm}$, eluted with $0.1 \mathrm{M} \mathrm{HOAc})$. The appropriate fractions were pooled and lyophilized to give compound 4 (acetate form, $1.85 \mathrm{~g}, 86 \%$ ).

${ }^{1} \mathrm{H}$ NMR (400 MHz, D 2 , r. t.) $\delta 4.86($ d, $1 \mathrm{H}, J=1.6 \mathrm{~Hz}, \mathrm{H}-1), 3.93$ (dd, $J=3.2 \mathrm{~Hz}$ and 1.6 Hz), $3.87(\mathrm{~m}, 1 \mathrm{H} 0,3.77-3.73(\mathrm{~m}, 3 \mathrm{H}), 3.64-3.62(\mathrm{~m}, 2 \mathrm{H}), 3.57(\mathrm{~m}, 2 \mathrm{H}), 3.0(\mathrm{t}$, $2 \mathrm{H}, J=8.0 \mathrm{~Hz}), 1.68(\mathrm{~m}, 4 \mathrm{H}), 1.46(\mathrm{~m}, 2 \mathrm{H})$.

${ }^{13} \mathrm{C}$ NMR (100 MHz, $\mathrm{D}_{2} \mathrm{O}$, r.t. $): \delta 99.2,72.3,70.2,69.6,67.0,66.3,60.5,26.1$. MALDI-TOF-MS: m/z 287.1 [M-H+Na]'.

\section{2-( $N$-Benzyloxycarbonyl)-aminoethyl $(2,3,4,6-$ tetra-O-benzoyl- $\beta$-D-} galactopyranosyl)-(1 $\rightarrow 4)$-2,3,6-tri-O-benzoyl- $\beta$-D-glucopyranoside (7)

Silver triflate $(768 \mathrm{mg}, 3.0 \mathrm{mmol})$ was added to a solution of 2-(Nbenzyloxycarbonyl)-aminoethanol 6 (665 $\mathrm{mg}, 3.4 \mathrm{mmol})$ and perbenzoyl lactosyl bromide $5(2.66 \mathrm{~g}, 2.3 \mathrm{mmol})$ in $\operatorname{dry} \mathrm{CH}_{2} \mathrm{Cl}_{2}(25 \mathrm{ml})$ at $-15^{\circ} \mathrm{C}$ containing $4-\AA \AA$ molecular sieves $(5 \mathrm{~g})$. After stirring for $30 \mathrm{~min}$., the mixture was allowed to reach room temperature over $30 \mathrm{~min}$. After 1h, TLC (toluene/acetone, 5:1) indicated that the reaction 
(®) 2004 American Chemical Society, Org. Lett., Gao ol040043a Supporting Info Page 5

was complete, the mixture was diluted with $\mathrm{CH}_{2} \mathrm{Cl}_{2}(60 \mathrm{ml})$, filtered through Celite, and washed with satd. aq. $\mathrm{NaHCO}_{3}$. The aqueous phase was extracted with $\mathrm{CH}_{2} \mathrm{Cl}_{2}(2 \times 30 \mathrm{ml})$, and the combined organic phases were dried (anhydrous $\mathrm{Na}_{2} \mathrm{SO}_{4}$ ), filtered, and concentrated. Flash chromatography of the residue on silica gel (toluene/acetone, 20:1) gave compound 7 (2.28 g, 78\%).

${ }^{1} \mathrm{H}$ NMR (400 MHz, $\mathrm{CDCl}_{3}$, r. t.) $\delta$ 7.88-8.01 (m, 12H, Aryl-H), 7.71-7.73 (m, 2H, Aryl-H), 7.64-7.12 (m, 26H, Aryl-H), 5.78 (t, 1H, $J=4.4 \mathrm{~Hz}, \mathrm{H}-3$ ), 5.71 (dd, 1H, $J=10.4$ $\mathrm{Hz}$ and $\left.7.8 \mathrm{~Hz}, \mathrm{H}-2^{\prime}\right), 5.40$ (dd, $1 \mathrm{H}, J=9.4 \mathrm{~Hz}$ and $6.0 \mathrm{~Hz}, \mathrm{H}-2$ ), $5.37(\mathrm{dd}, 1 \mathrm{H}, J=10.4 \mathrm{~Hz}$ and $\left.3.2 \mathrm{~Hz}, \mathrm{H}-3^{\prime}\right), 5.10(\mathrm{t}, 1 \mathrm{H}), 4.95(\mathrm{~m}, 1 \mathrm{H}), 4.89\left(\mathrm{~d}, 1 \mathrm{H}, J=8.0 \mathrm{~Hz}, \mathrm{H}-1^{\prime}\right), 4.66(\mathrm{~d}, 1 \mathrm{H}$, $J=8.0 \mathrm{~Hz}, \mathrm{H}-1$ ), 4.60 (dd, $1 \mathrm{H}, J=12.0 \mathrm{~Hz}$ and $1.6 \mathrm{~Hz}, \mathrm{H}-6 \mathrm{a}), 4.47(\mathrm{dd}, 1 \mathrm{H}, J=12.0 \mathrm{~Hz}$ and $4.4 \mathrm{~Hz}, \mathrm{H}-6 \mathrm{~b}), 4.23(\mathrm{t}, 1 \mathrm{H}, \mathrm{H}-4), 3.91,3.76\left(2 \mathrm{~m}, 2 \mathrm{H}, \mathrm{OCH}_{2}\right), 3.80(\mathrm{~m}, 2 \mathrm{H}, \mathrm{H}-5$ and H-6), 3.73-3.66 (m, 2H, H-6a' and H-6b'), 3.30 (m, 2H, $\left.\mathrm{CH}_{2} \mathrm{NH}\right)$.

${ }^{13} \mathrm{C} \cdot \mathrm{NMR}\left(100 \mathrm{MHz}, \mathrm{CDCl}_{3}\right.$ r. t.): $\delta 165.9,165.6,165.4,165.3,165.22,164.8(7 \mathrm{CO})$, 156.3 (CONH), 136.6, 133.5, 133.4, 133.3, 130.0, 129.8, 129.7, 129.6, 129.5, 129.4, $129.1,129.0,128.8,128.7,128.6,128.5,128.4,128.3,128.0$ (Aryl-C), 101.42, 100.98 (C-1 and C-1'), 76.7, 75.9, 73.1, 72.6, 71.76, 71.78, 71.4, 69.9, 67.5, 66.5, 62.2, 61.1, 40.8.

FAB-MS m/z: $1248[\mathrm{M}+\mathrm{H}]^{+}$; FAB-HRMS: calcd for $[\mathrm{M}+\mathrm{H}]^{+} \mathrm{C}_{71} \mathrm{H}_{62} \mathrm{O}_{20} \mathrm{~N}$, 1248.3864; found, 1248.3880 .

\section{2-Aminoethyl $\beta$-D-galactopyranosyl-( $\rightarrow 4$ )-O- $\beta$-D-glucopyranoside (8)}

The fully protected lactoside $7(3.5 \mathrm{~g}, 2.8 \mathrm{mmol})$ was taken up in dry methanol (60 $\mathrm{ml}$ ), and the solution was made basic ( $\mathrm{pH} 11$ towards moist $\mathrm{pH}$ paper) by the addition of 
$\mathrm{NaOMe}$, and the mixture was left at room temperature. After TLC indicated completion of the reaction $(6 \mathrm{~h}), \mathrm{H}_{2} \mathrm{O}(20 \mathrm{ml})$ was added to dissolve precipitates, and the reaction was left to stir for a further $2 \mathrm{~h}$. The reaction was neutralized with Dowex-50WX8-400 $\left(\mathrm{H}^{+}\right.$ form) and filtered. The filtrate and washings were concentrated to $15 \mathrm{ml}$, and then acetic acid $(10 \mathrm{ml})$ and $\mathrm{Pd} / \mathrm{C} 10 \%(500 \mathrm{mg})$ were added, whereafter the mixture was hydrogenated at atmosphere pressure for $12 \mathrm{~h}$. When the reaction was complete (by TLC chloroform/methanol, 1:1), the mixture was filtered through a pad of Celite, washed with water $(30 \mathrm{ml}$ ), concentrated and lyophilized to give compound $\mathbf{8}$ (acetate form, $1.02 \mathrm{~g}$, $82 \%)$.

${ }^{1} \mathrm{H}$ NMR $\left(400 \mathrm{MHz}, \mathrm{D}_{2} \mathrm{O}\right.$, r. t.) $\delta 4.54$ (d, $\left.1 \mathrm{H}, \mathrm{H}-1, J=7.6 \mathrm{~Hz}\right), 4.45$ (d, $1 \mathrm{H}, J=8.0 \mathrm{~Hz}$, H-1'), $4.12(\mathrm{~m}, 1 \mathrm{H}), 3.96(\mathrm{~m}, 2 \mathrm{H}), 3.92,3.76\left(2 \mathrm{~m}, 2 \mathrm{H}, \mathrm{OCH}{ }_{2}\right), 3.84-3.62(\mathrm{~m}, 8 \mathrm{H}), 3.55$ (m, $1 \mathrm{H}), 3.37(\mathrm{~m}, 1 \mathrm{H}), 3.25\left(\mathrm{t}, 2 \mathrm{H}, \mathrm{CH}_{2} \mathrm{NCO}\right)$.

${ }^{13} \mathrm{C} \cdot \mathrm{NMR}\left(100 \mathrm{MHz}, \mathrm{D}_{2} \mathrm{O}\right.$, r. t.): $\delta 102.5,101.5$ (C-1,1'), 77.80, 75.0, 74.3, 73.8, 72.3, $72.1,70.5,68.1,65.6,60.6,59.5,39.0$.

FAB-MS m/z: $386[\mathrm{M}+\mathrm{H}]^{+}$; FAB-HRMS Calcd for $\mathrm{C}_{14} \mathrm{H}_{28} \mathrm{NO}_{11}[\mathrm{M}+\mathrm{H}]^{+}, 386.1660$; found, 386.1664 .

\section{$N$-(9-Fluorenylmethoxycarbonyl)-aminohexyl (2,3,4,6-tetra-O-benzoyl- $\beta$-D-} galactopyranosyl)-(1 $\rightarrow 4)-2,3,6$-tri-O-benzoyl- $\beta$-D-glucopyranoside (10)

Solid Silver triflate $(429 \mathrm{mg}, 1.68 \mathrm{mmol})$ was added to a solution of N-(9fluorenylmethoxycarbonyl)-aminohexyl-1-ol 9 at $-15^{\circ} \mathrm{C}(608 \mathrm{mg}, 1.79 \mathrm{mmol})$ and perbenzoyl lactosyl bromide $5(1.36 \mathrm{~g}, 1.2 \mathrm{mmol})$ in dry $\mathrm{CH}_{2} \mathrm{Cl}_{2}(15 \mathrm{ml})$ containing $4 \AA$ molecular sieves $(2.0 \mathrm{~g})$. After stirring for $30 \mathrm{~min}$., the mixture was allowed to warm to 
@ 2004 American Chemical Society, Org. Lett., Gao ol040043a Supporting Info Page 7

room temperature during $30 \mathrm{~min}$, and $2 \mathrm{~h}$ thereafter, the mixture was diluted with $\mathrm{CH}_{2} \mathrm{Cl}_{2}$ $(50 \mathrm{ml})$, filtered through a pad of Celite, and washed with satd. aq. $\mathrm{NaHCO}_{3}(50 \mathrm{ml})$. The aqueous phase was extracted with $\mathrm{CH}_{2} \mathrm{Cl}_{2}(2 \times 30 \mathrm{ml})$, and the combined organic phases were dried (anhydrous $\mathrm{Na}_{2} \mathrm{SO}_{4}$ ), filtered, and concentrated. Flash column chromatography (toluene/acetone, 20:1) of the residue gave compound $10(1.23 \mathrm{~g}, 74 \%)$.

${ }^{1} \mathrm{H}$ NMR $\left(400 \mathrm{MHz}, \mathrm{CDCl}_{3}\right.$, r. t.) 8 7.89-8.03 (m, 12H, Aryl-H), 7.77-7.72 (m, 4H, Aryl-H), 7.64-7.11 (m, 27H, Aryl-H), 5.80 (t, 1H, $J=9.4$ Hz, H-3), 5.72 (dd, 1H, $J=10.4$ $\mathrm{Hz}$ and $\left.7.8 \mathrm{~Hz}, \mathrm{H}-2^{\prime}\right), 5.46(\mathrm{dd}, 1 \mathrm{H}, J=10.0 \mathrm{~Hz}$ and $8.0 \mathrm{~Hz}, \mathrm{H}-2), 5.38(\mathrm{dd}, 1 \mathrm{H}, J=10.4 \mathrm{~Hz}$ and $\left.3.2 \mathrm{~Hz}, \mathrm{H}-3^{\prime}\right), 4.87$ (d, 1H, $\left.J=8.0 \mathrm{~Hz}, \mathrm{H}-1^{\prime}\right), 4.67$ (d, 1H, $J=8.0 \mathrm{~Hz}, \mathrm{H}-1$ ), 4.60 (dd, 1 $\mathrm{H}, J=12.0 \mathrm{~Hz}$ and $1.6 \mathrm{~Hz}, \mathrm{H}-6 \mathrm{a}), 4.48(\mathrm{dd}, 1 \mathrm{H}, J=12.0 \mathrm{~Hz}$ and $4.4 \mathrm{~Hz}, \mathrm{H}-6 \mathrm{~b}), 4.39$ (dd, 2 $\left.\mathrm{H}, \mathrm{OCOCH}_{2}\right), 4.25\left(\mathrm{~m}, 2 \mathrm{H}, \mathrm{H}-4, \mathrm{OCOCH}_{2} \mathrm{CH}\right), 3.86\left(\mathrm{~m}, 2 \mathrm{H}, \mathrm{H}-5\right.$ ' and $\left.\mathrm{OCH}_{2}\right), 3.80(\mathrm{~m}, 1$ H, H-5), 3.70 (dd, 2H, $J=6.4 \mathrm{~Hz}$ and $3.6 \mathrm{~Hz}, \mathrm{H}-6 \mathrm{a}$ 'and H-6b'), 3.43 (m, 1H), 2.98 (m, 2H, $\left.\mathrm{CH}_{2} \mathrm{NHCO}\right), 1.46(\mathrm{~m}, 2 \mathrm{H}), 1.18-1.11(\mathrm{~m}, 6 \mathrm{H})$.

${ }^{13} \mathrm{C}$ NMR (100 MHz, $\mathrm{CDCl}_{3}$ r. t.): $\delta 165.9,165.6,165.4,165.2,165.1,164.8$ (7 CO), 156.3(CONH), 144.0, 141.3, 133.6, 133.4, 133.3, 133.2, 130.0, 129.8, 129.7, 129.6, 129.5, $129.4,129.0,128.8,128.7,128.64,128.58,128.52,128.4,128.3,127.8,127.0,125.3$, 125.0, 120.0 (Aryl-C), 101.19, 100.98 (C-1 and C-1'), 76.1, 73.0, 72.9, 71.8, 71.4, 70.1, $69.9,67.5,66.4,62.4,61.1,47.3,40.8,29.6,29.1,26.1,25.4$.

FAB-MS: $\mathrm{m} / \mathrm{z}[\mathrm{M}+\mathrm{H}]^{+} 1392.7$.

\section{6-Aminohexyl $\beta$-D-galactopyranosyl-(1 $\rightarrow 4)$-O- $\beta$-D-glucopyranoside (11)}

$\mathrm{NaOMe}(740 \mathrm{mg})$ was added to a stirred solution of fully protected lactoside 10 (3.8 $\mathrm{g})$ in dry methanol $(50 \mathrm{ml})$ and the mixture was left at room temperature. After the 
( 2004 American Chemical Society, Org. Lett., Gao ol040043a Supporting Info Page 8

reaction was complete (by TLC) $(8 \mathrm{~h}), \mathrm{H}_{2} \mathrm{O}(25 \mathrm{ml})$ was added, and the reaction was stirred for a further $1 \mathrm{~h}$, during which time the precipitate dissolved. The reaction was neutralized with Dowex-50WX8-400 $\left(\mathrm{H}^{+}\right.$form $)$and filtered. The solvents were removed in vacuo and the resulting residue was taken up with $\mathrm{H}_{2} \mathrm{O}(50 \mathrm{ml})$, and extracted with toluene $(30 \mathrm{ml})$ and ethyl acetate $(30 \mathrm{ml})$. The aqueous layer was evaporated to a small volume $(5 \mathrm{ml})$ and applied to a Sephadex G-25 column $(5 \times 200 \mathrm{~cm}, 0.1 \mathrm{M}$ HOAc as eluent). Appropriate fractions were pooled, concentrated to a small volume and lyophilized to give the fully deblocked compound 11 (acetate form, $1.14 \mathrm{~g}, 95 \%$ ).

${ }^{1} \mathrm{H}$ NMR (400 MHz, $\mathrm{D}_{2} \mathrm{O}$, r. t.): $\delta 4.48$ (d, $\left.1 \mathrm{H}, J=7.6 \mathrm{~Hz}, \mathrm{H}-1\right), 4.44$ (d, $1 \mathrm{H}, J=8.0 \mathrm{~Hz}$, $\left.\mathrm{H}-1^{\prime}\right), 3.96(\mathrm{~m}, 2 \mathrm{H}), 3.91,3.76\left(2 \mathrm{~m}, 2 \mathrm{H}, \mathrm{OCH}_{2}\right), 3.82-3.78(\mathrm{~m}, 2 \mathrm{H}), 3.73-3.63(\mathrm{~m}, 6 \mathrm{H})$, , found, 442.2285. 3.60-3.52 (m, 2H), $3.30(\mathrm{~m}, 1 \mathrm{H}), 2.99\left(\mathrm{t}, 2 \mathrm{H}, \mathrm{CH} \mathrm{H}_{2} \mathrm{NCO}\right), 1.65(\mathrm{~m}, 4 \mathrm{H})$, $1.41(\mathrm{~m}, 4 \mathrm{H})$.

${ }^{13} \mathrm{C}$ NMR (100 MHz, D 2 O, r. t.): $\delta 102.5,101.6\left(\mathrm{C}-1,1^{\prime}\right), 78.0,74.9,74.3,74.0,72.4$, $72.1,70.5,69.9,68.1,60.6,59.7,38.9,28.0,26.2,24.8,24.1$.

FAB-MS m/z: $442[\mathrm{M}+\mathrm{H}]^{+}$; HRMS (FAB) Calcd for $\mathrm{C}_{18} \mathrm{H}_{36} \mathrm{NO}_{11}[\mathrm{M}+\mathrm{H}]^{+}, 442.2288$.

\section{Synthesis of Mercaptans 12, 13, 14 from the Corresponding Primary Amines.}

General Procedures ${ }^{4}$. The $\mathrm{RNH}_{2}(2 \mathrm{mmol})$, DTT (5 mmol) and thiobutyrolactone (12 $\mathrm{mmol})$ were added to a mixture of $0.5 \mathrm{M}$ sodium bicarbonate $(12 \mathrm{ml})$ and ethanol $(12 \mathrm{ml})$. A stream of $\mathrm{N}_{2}$ was bubbled through the solution for $15 \mathrm{~min}$. Then the mixture was heated at $50^{\circ} \mathrm{C}$ for $8--10 \mathrm{~h}$ until TLC indicated the reation was complete. The mixture was cooled to room temperature and then adjusted to $\mathrm{pH} 7$ with $1 \mathrm{M} \mathrm{HCl}$. The solvents and volatile side products were removed in vacuo, and the residue was subjected to 
@ 2004 American Chemical Society, Org. Lett., Gao ol040043a Supporting Info Page 9

chromatograpy on silica gel.

5-(3'-Mercaptopropionylamido)pentyl $\alpha$-D- mannopyranoside (12) from (4): Reflux for $8 \mathrm{~h}$, eluent, ethyl acetate/methanol 3:1; yield, $90 \%$.

${ }^{1} \mathrm{H}$ NMR $\left(400 \mathrm{MHz}, \mathrm{D}_{2} \mathrm{O}\right.$, r. t.) $\delta 4.85(\mathrm{~s}, 1 \mathrm{H}, \mathrm{H}-1), 3.92(\mathrm{~m}, 1 \mathrm{H}), 3.89-3.86(\mathrm{~m}, 1 \mathrm{H})$, 3.79-3.76 (m, 2H), 3.74-3.71 (m, 1H), 3.64-3.62 (m, 2H), $3.53(\mathrm{~m}, 1 \mathrm{H}), 3.19(\mathrm{t}, 2 \mathrm{H}$, $\mathrm{CH}_{2} \mathrm{NH}$ ), 2.54 (t, $2 \mathrm{H}, \mathrm{CH}_{2} \mathrm{SH}$ ), 2.35 (t, $2 \mathrm{H}, \mathrm{COCH}_{2}$ ), 1.88 (quintet, $2 \mathrm{H}, \mathrm{CH}_{2} \mathrm{CH}_{2} \mathrm{CH}_{2} \mathrm{SH}$ ), $1.62(\mathrm{~m}, 2 \mathrm{H}), 1.53(\mathrm{~m}, 2 \mathrm{H}), 1.38(\mathrm{~m}, 2 \mathrm{H})$.

${ }^{13} \mathrm{C}$ NMR $\left(100 \mathrm{MHz}, \mathrm{D}_{2} \mathrm{O}\right.$, r. t.): $\delta 175.34,99.20,72.29,70.20,69.64,67.18,66.30$, $60.47,38.72,33.99,29.11,27.72,27.64,22.64,22.40$.

MALDI-TOF MS: m/z $366.2[\mathrm{M}-\mathrm{H}]^{*}$.

2-(3'-Mercaptopropionylamido)ethyl $\beta$-D-galactopyranosyl-(1 $\rightarrow 4)-0-\beta-D-$

glucopyranoside (13) from (8): Reflux for $10 \mathrm{~h}$, eluent, ethyl acetate/methanol 2:1; yield, $86 \%$.

${ }^{1} \mathrm{H}$ NMR (400 MHz, $\mathrm{D}_{2} \mathrm{O}$, r. t.) $\delta 4.49$ (d, $\left.1 \mathrm{H}, J=7.6 \mathrm{~Hz}\right), 4.45$ (d, $\left.1 \mathrm{H}, J=8.0 \mathrm{~Hz}\right), 3.99$ $3.97(\mathrm{~m}, 2 \mathrm{H}), 3.92(\mathrm{~d}, 1 \mathrm{H}, \mathrm{J}=3.2 \mathrm{~Hz}), 3.83-3.73(\mathrm{~m}, 4 \mathrm{H}), 3.68-3.64(\mathrm{~m}, 3 \mathrm{H}), 3.60(\mathrm{~m}, 1 \mathrm{H})$, 3.47-3.42 (m, 2H), $2.55\left(\mathrm{t}, 2 \mathrm{H}, \mathrm{CH}_{2} \mathrm{SH}\right.$ ), 2.38 (t, $2 \mathrm{H}, \mathrm{COCH}_{2}$ ), 1.89 (quintet, $2 \mathrm{H}$, $\left.\mathrm{CH}_{2} \mathrm{CH}_{2} \mathrm{SH}\right)$.

${ }^{13} \mathrm{C}$ NMR (100MHz, $\mathrm{D}_{2} \mathrm{O}$, r. t.): $\delta 175.84(\mathrm{CONH}), 102.48,101.73$ (C-1,1'), 77.93, $74.89,74.30,73.83,72.35,72.04,70.48,68.08,67.97,60.56,59.63,38.80,33.93,28.96$, 22.61 .

MALDI-TOF-MS: m/z $510.2[\mathrm{M}+\mathrm{Na}]^{+}$. 
6-(3'-Mercaptopropionylamido)hexyl- $\beta$-D-galactopyranosyl-(1 $\rightarrow 4)-0-\beta-D-$

glucopyranoside (14) from (11): Reflux for $10 \mathrm{~h}$, eluent, ethyl acetate/methanol 2:1; yield, $82 \%$.

${ }^{1} \mathrm{H}$ NMR (400 MHz, D $2 \mathrm{O}$, r. t.) $\delta 4.48\left(\mathrm{~d}, 1 \mathrm{H}, J=8.0 \mathrm{~Hz}, \mathrm{H}-1^{\prime}\right), 4.45(\mathrm{~d}, 1 \mathrm{H}, J=8.0 \mathrm{~Hz}$, $\mathrm{H}-1), 3.99-3.90(\mathrm{~m}, 3 \mathrm{H}), 3.82-3.76(\mathrm{~m}, 3 \mathrm{H}), 3.73-3.70(\mathrm{~m}, 1 \mathrm{H}), 3.68-3.63(\mathrm{~m}, 4 \mathrm{H}), 3.60-$ $3.52(\mathrm{~m}, 2 \mathrm{H}), 3.30(\mathrm{~m}, 1 \mathrm{H}), 3.18\left(\mathrm{t}, 2 \mathrm{H}, \mathrm{CH}_{2} \mathrm{NH}\right), 2.54\left(\mathrm{t}, 2 \mathrm{H}, \mathrm{CH}_{2} \mathrm{SH}\right), 2.35(\mathrm{t}, 2 \mathrm{H}$, $\mathrm{COCH}_{2}$ ), 1.88 (quintet, $2 \mathrm{H}, \mathrm{COCH}_{2} \mathrm{CH}_{2} \mathrm{CH}_{2} \mathrm{SH}$ ) $1.63,1.51,1.34(3 \mathrm{~m}, 8 \mathrm{H})$.

${ }^{13} \mathrm{C}$ NMR (100MHz, D 2 O, r. t.) $\delta 175.40$ (CONH), 102.47, 101.58 (C-1,1'), 77.92, $74.90,74.30,74.01,72.40,72.07,70.50,70.12,68.10,60.58,59.65,38.78,34.01,29.13$, $28.18,27.69,25.29,24.22,22.62$.

MALDI-TOF MS: m/z $565.3[\mathrm{M}-\mathrm{H}+\mathrm{Na}]^{-}$.

\section{Glycocluster (15)}

A solution of thiol $12(528 \mathrm{mg}, 1.45 \mathrm{mmol})$ in $\mathrm{H}_{2} \mathrm{O}(10 \mathrm{ml})$ was mixed with a solution of octavinyl-POSS (26 mg, $0.041 \mathrm{mmol})$ in THF (10 ml) was added. A stream of nitrogen was bubbled through the mixture for $30 \mathrm{~min}$. AIBN (48 $\mathrm{mg}, 0.29 \mathrm{mmol}$ ) was added. The suspension, kept under $\mathrm{N}_{2}$, was irradiated at $254 \mathrm{~nm}$ and stirred for $24 \mathrm{~h} .{ }^{1} \mathrm{H}$ NMR indicated completion of the reaction. Following the removal of the solvents, the residue was purified by gel filtration chromatography (Sepadex G-15, 2x145 cm, $\mathrm{H}_{2} \mathrm{O}$ as eluent) to afford the product 15 as a white foam (103 $\mathrm{mg}, 70 \%)$.

${ }^{1} \mathrm{H}$ NMR (400 MHz, D $2 \mathrm{O}$, r. t.) $\delta 4.85(\mathrm{~s}, 8 \mathrm{H}, \mathrm{H}-1), 3.91(\mathrm{~m}, 8 \mathrm{H}), 3.89-3.85(\mathrm{~m}, 8 \mathrm{H})$, 3.80-3.66 (m, 32H), 3.64-3.61 (m, 8H), $3.52(\mathrm{~m}, 8 \mathrm{H}), 3.20(\mathrm{bs}, 16 \mathrm{H}), 2.70(\mathrm{bs}, 16 \mathrm{H}), 2.36$ (bs, 16H), $1.90(\mathrm{bs}, 16 \mathrm{H}), 1.63(\mathrm{bs}, 16 \mathrm{H}), 1.55$ (bs, 16H), 1.41 (bs, 16H), 1.09 (bs, 16H, $\mathrm{SiCH}_{2}$ ). 
๔ 2004 American Chemical Society, Org. Lett., Gao ol040043a Supporting Info Page 11

${ }^{13} \mathrm{C}$ NMR (100 MHz, D2 O, r. t.) $\delta 173.98$ (8 CONH), 99.34 ( C-1), 72.32, 70.35, 69.82, $67.04,66.25,60.48,38.91,34.50,28.15,25.07,22.78,12.13$.

MALDI-TOF MS (DHB-HIQ matrix): $3608.30[\mathrm{M}+\mathrm{K}]^{+}$

\section{Glycocluster (16)}

Reaction of thiol 13 ( $385 \mathrm{mg}, 0.79 \mathrm{mmol})$ with octavinyl-POSS (16 mg, $0.025 \mathrm{mmol})$ and AIBN (28 mg, $0.17 \mathrm{mmol})$ in a mixture of $\mathrm{H}_{2} \mathrm{O}: \operatorname{THF}(1: 1,12 \mathrm{ml})$ for $48 \mathrm{~h}$ as described for (15) afforded 16 as a white foam (75 $\mathrm{mg}, 66 \%)$.

${ }^{1} \mathrm{H}$ NMR (400 MHz, $\mathrm{D}_{2} \mathrm{O}$, r. t.) $\delta 4.49$ (d, $8 \mathrm{H}, J=8.0 \mathrm{~Hz}, \mathrm{H}-1$ '), 4.45 (d, $8 \mathrm{H}, J=7.6 \mathrm{~Hz}$, $\mathrm{H}-1), 3.99-3.92(\mathrm{~m}, 24 \mathrm{H}), 3.83-3.72(\mathrm{~m}, 40 \mathrm{H}), 3.68-3.65(\mathrm{~m}, 24 \mathrm{H}), 3.62-3.53(\mathrm{~m}, 16 \mathrm{H})$, 3.46 (m, 16H), 3.35 (bt, 8 H), 2.70 (bs, 16H), 2.60 (bs, 16H), 2.38 (bs, 16H), 1.90 (bs, 16 H), 1.06 (bs, 16H, $\mathrm{SiCH}_{2}$ ).

${ }^{13} \mathrm{C}$ NMR (100 MHz, D 2 O, r. t.) $\delta 175.30(8 \mathrm{CONH}), 102.55,101.85$ ( $8 \mathrm{C}-1$ and $8 \mathrm{C}-$ 1'), $78.00,74.93,74.36,73.89,72.38,72.12,70.52,68.11,60.60,59.71,38.89,34.34$, 24.76, 12.51 .

MALDI-TOF MS (DHB-HIQ matrix): $4568.50[\mathrm{M}+\mathrm{K}]^{+}$.

\section{Glycocluster (17)}

Reaction of thiol $11(588 \mathrm{mg}, 1.08 \mathrm{mmol})$ and octavinyl-POSS (22 mg, $0.035 \mathrm{mmol})$ with AIBN (36 mg, $0.22 \mathrm{mmol})$ in $\mathrm{H}_{2} \mathrm{O} / \mathrm{THF}(1: 1,16 \mathrm{ml})$ for $36 \mathrm{~h}$ as described for 15 gave the product 17 as a foam $(128 \mathrm{mg}, 73 \%)$.

'H NMR (400 MHz, D $2 \mathrm{O}$, r. t.): $\delta 4.47$ (bs, 8H, H-l'), 4.45 (bs, 8H, H-1), 3.98-3.89 (m, 24H), 3.84-3.76 (m, 24H), 3.74-3.71(m, 8H), 3.69-3.62 (m, 32H), 3.58-3.54 (m, 16H), 3.32 (bt, 8H), 3.19 (bs, 16H), 2.69 (bs, 16H), 2.59 (bs, 16H), 2.36 (bs, 16H), 1.89 (bs, 
16H), 1.64 (bs, 16H), 1.53 (bs, 16H), 1.38 (bs, 32H), 1.08 (bs, 16H, SiCH $H_{2}$.

${ }^{13} \mathrm{C} \mathrm{NMR}\left(100 \mathrm{MHz}, \mathrm{D}_{2} \mathrm{O}\right.$, r. t.) $\delta 173.99$ (CONH), 102.56, 101.82 (C-1 and C-1'), $78.04,74.93,74.33,74.10,72.45,72.16,70.53,69.95,68.12,60.60,59.77,38.96,34.53$, $28.57,24.63,12.15$

MALDI-TOF MS (DHB-HIQ matrix): $5016.80[\mathrm{M}+\mathrm{K}]^{+}$

\section{Inhibitory Effect of Glycoclusters on the Binding between RCA120 and}

\section{Asialooligasaccharides Derived from Human a1-acid Glycoprotein}

We examined the binding of 16 or 17 by RCA120 which is specific for $\beta$-linked galactose at the non-reducing termini of oligosaccharides. The studies were performed by measuring the inhibitory activity of 16 or 17 on the binding of asialooligasaccharides derived from human $\alpha 1$-acid Glycoprotein (AGP) by RCA120 using capillary affinity electrophoresis (CAE) ${ }^{5}$

Procedures for capillary affinity electrophoresis (CAE) CAE was performed using a P/ACE MDQ glycoprotein system (Beckman Coulter, Fullerton, CA, USA) equipped with a eCAP N-CHO capiliary $(20 \mathrm{~cm}$ effective length, $30 \mathrm{~cm}$ total length, $50 \mu \mathrm{m}$ i.d., Beckman Coulter) using an argon-laser induced fluorescence detector as described previously ${ }^{5}$. Detection was performed by installing a $520 \mathrm{~nm}$ filter for emission with a $488 \mathrm{~nm}$ argon-laser for excitation. Tris-acetate buffer (100 mM, pH 7.4) was used as electrolyte throughout the work. The sample solution was introduced to the capillary by pressure method $(0.5 \mathrm{psi}, 5 \mathrm{sec})$. Separation was performed at $25^{\circ} \mathrm{C}$ at the applied potential of $18 \mathrm{kV}$, and data were collected and analyzed with a standard 32 Karat software (Version 4.0, Beckman Coulter) on Microsoft Windows 2000. Briefly, prior to CAE, a mixture of 1-aminopyrene-3, 6, 8-trisulfonate (APTS)-labled 
oligosaccharides was electrophoresed in the absence of lectin. Then, the same electrolyte containing a lectin at the specified concentration was filled in the capillary, and the same mixture of fluorescent oligosaccharides was analyzed.

An example using 16 as an inhibitor is shown in Fig. 1

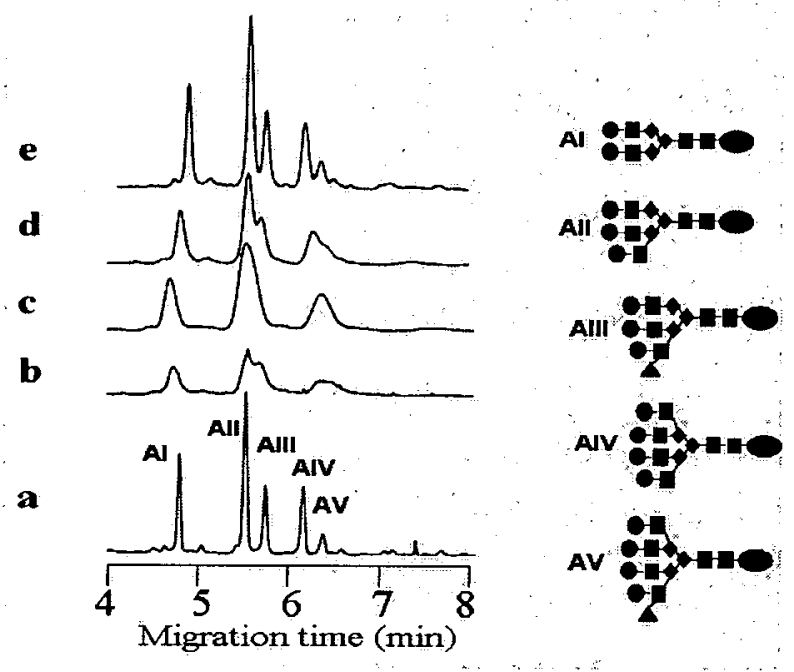

Fig.1 Inhibitory effect of 16 on the binding between RCA120 and APTS-labeled asialooligosaccharides from human $\alpha 1$-acid glycoprotein.

(a) Separation of APTS-labeled asialooligosaccharides from human AGP; (b) Separation of the same mixture in the presence of RCA120 at $0.25 \mu \mathrm{M}$; (c) 16 was added to the mixture at $100 \mathrm{nM}$; (d) 16 was added to the mixture at $1 \mu \mathrm{M}$; (e) 16 was added to the mixture at $10 \mu \mathrm{M}$. Analytical conditions and fluorescent labeling of oligosaccharides were described in ref. 5 .

AGP contains di-, tri- and tetra-antennary carbohydrate chains, and the asialooligosaccharides thereof have common Galp1-4GlcNAc branches and show high affinity toward RCA120. In the absence of 16, all oligosaccharides showed obvious retardation and peak broadening due to the binding reaction when analyzed by capillary electrophoresis in the electrolyte containing RCA120 even at $0.25 \mu \mathrm{M}$ concentration (Fig. $\mathbf{1}, \mathbf{a}$ and $\mathbf{b})$. Addition of $\mathbf{1 6}$ to the electrolyte clearly resulted inhibition of the binding. At $10 \mu \mathrm{M}$ of 16 , the electropherogram showed almost the same migrations with that observed in the absence of 16, and indicated complete inhibition of the binding (Fig.1, e). 
We also examined binding effect of $\mathbf{1 7}$ and found similar affinity toward RCA120. In contrast, the binding was not fully inhibited even at $20 \mathrm{mM}$ lactose in the electrolyte (data not shown). These data indicated that 16 and 17 showed 200 times or higher inhibitory effect than lactose, and such high affinity is probably due to clustering effect. As predicted by binding specificity of RCA120,15, which contains only mannose residues did not show any binding at the concentration of $10 \mu \mathrm{M}$, where 16 and 17 showed complete inhibition of the binding toward asialooligosaccharides. The detailed binding characteristics will be reported elsewhere (manuscript in preparation).

\section{References}

(1) Hirai, Y.; Nagatsu, M. Chemistry Lett. 1994, 21-22.

(2) Lin, P. Kong Thoo; Kuksa, V. A.; Maguire, N. M. Synthesis. 1998, 6, 859-866.

(3) Ichikawa, Y; Lee, Y. C. Carbohydr. Res. 1990, 198, 235-246.

(4) Norberg, T.; Blixt, O. J. Carbohydr. Chem., 1997, 16(2), 143-154.

(5) Nakajima, K.; Oda, Y.; Kinoshita, M.; Kakehi, K. J. Proteome Res. 2003, 2, 81-88 
(c) 2004 American Chemical Society, Org. Lett., Gao ol040043a Supporting Info Page 15

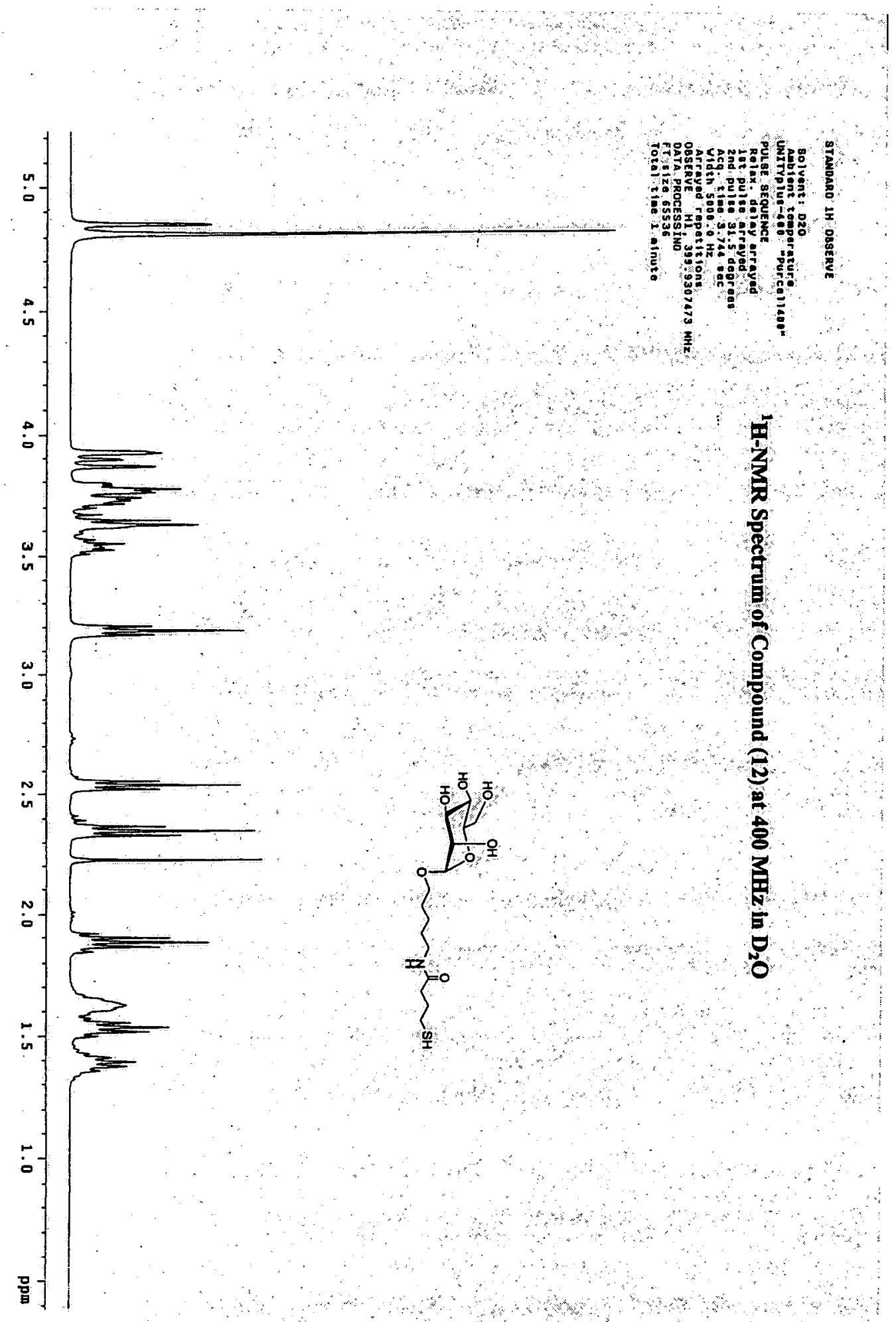


(C) 2004 American Chemical Society, Org. Lett., Gao ol040043a Supporting Info Page 16
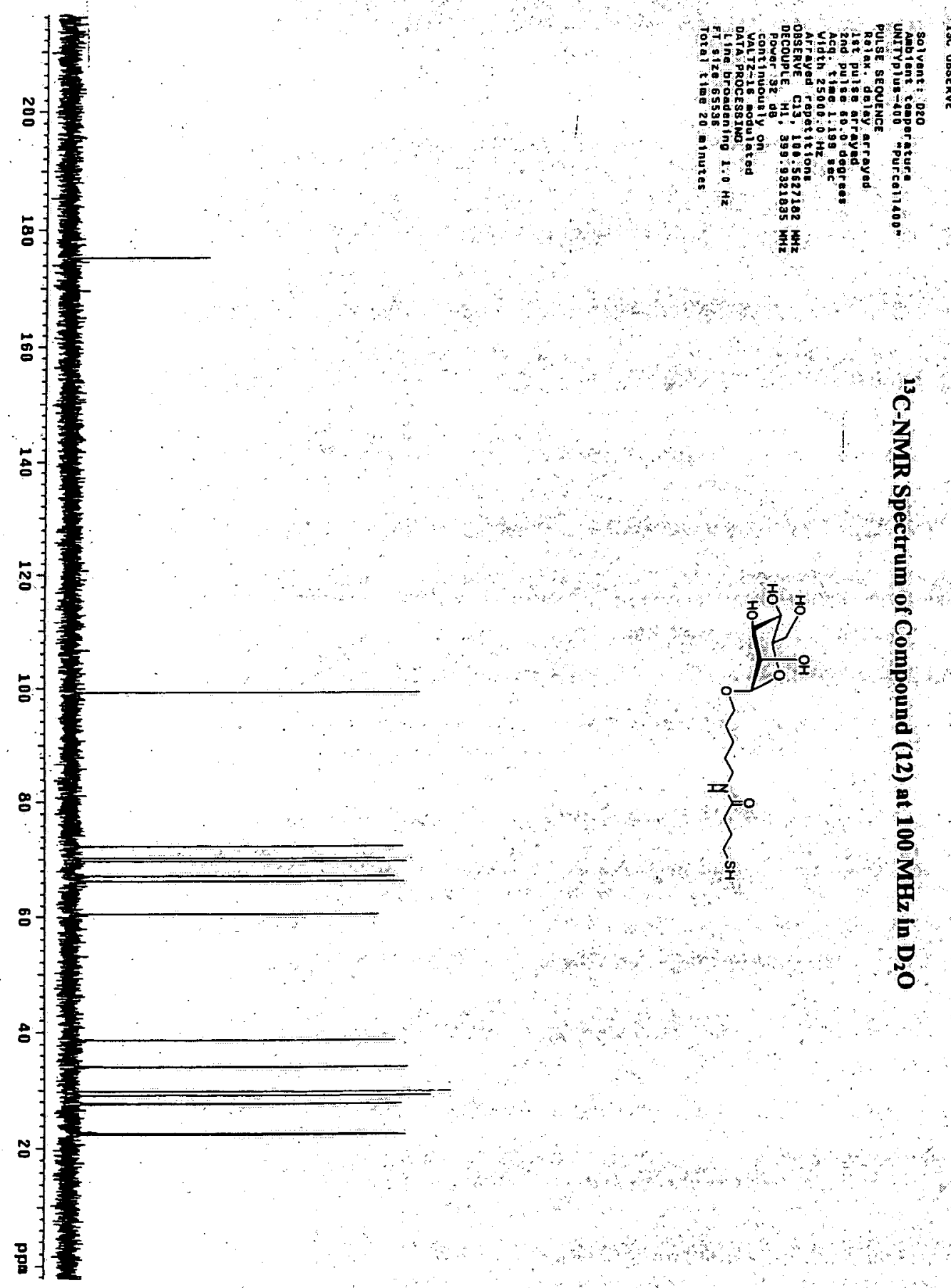

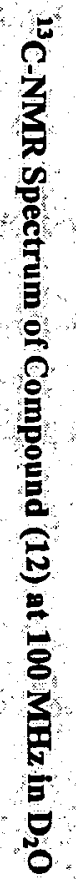


(C) 2004 American Chemical Society, Org. Lett., Gao ol040043a Supporting Info Page 17

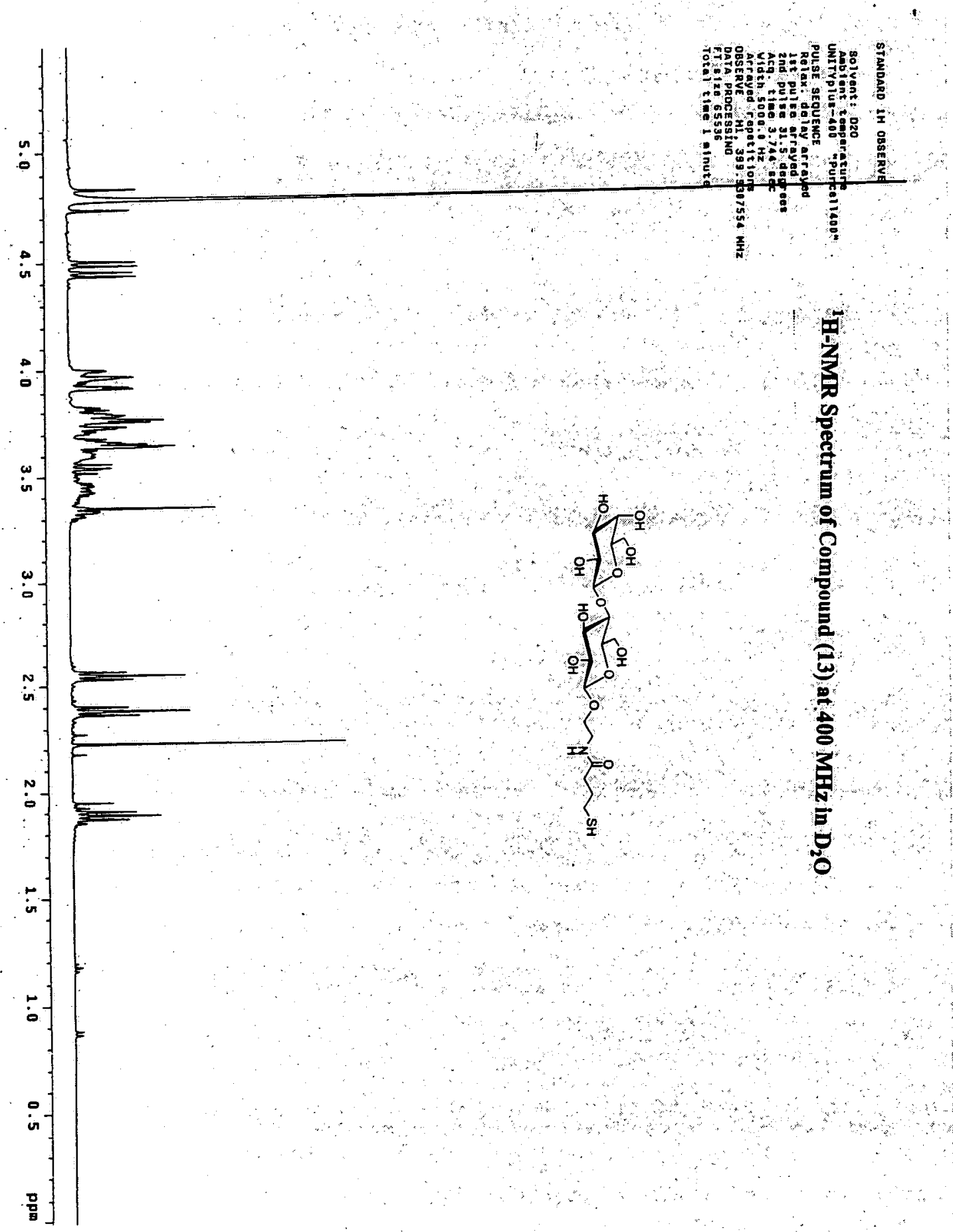


(C) 2004 American Chemical Society, Org. Lett., Gao ol040043a Supporting Info Page 18
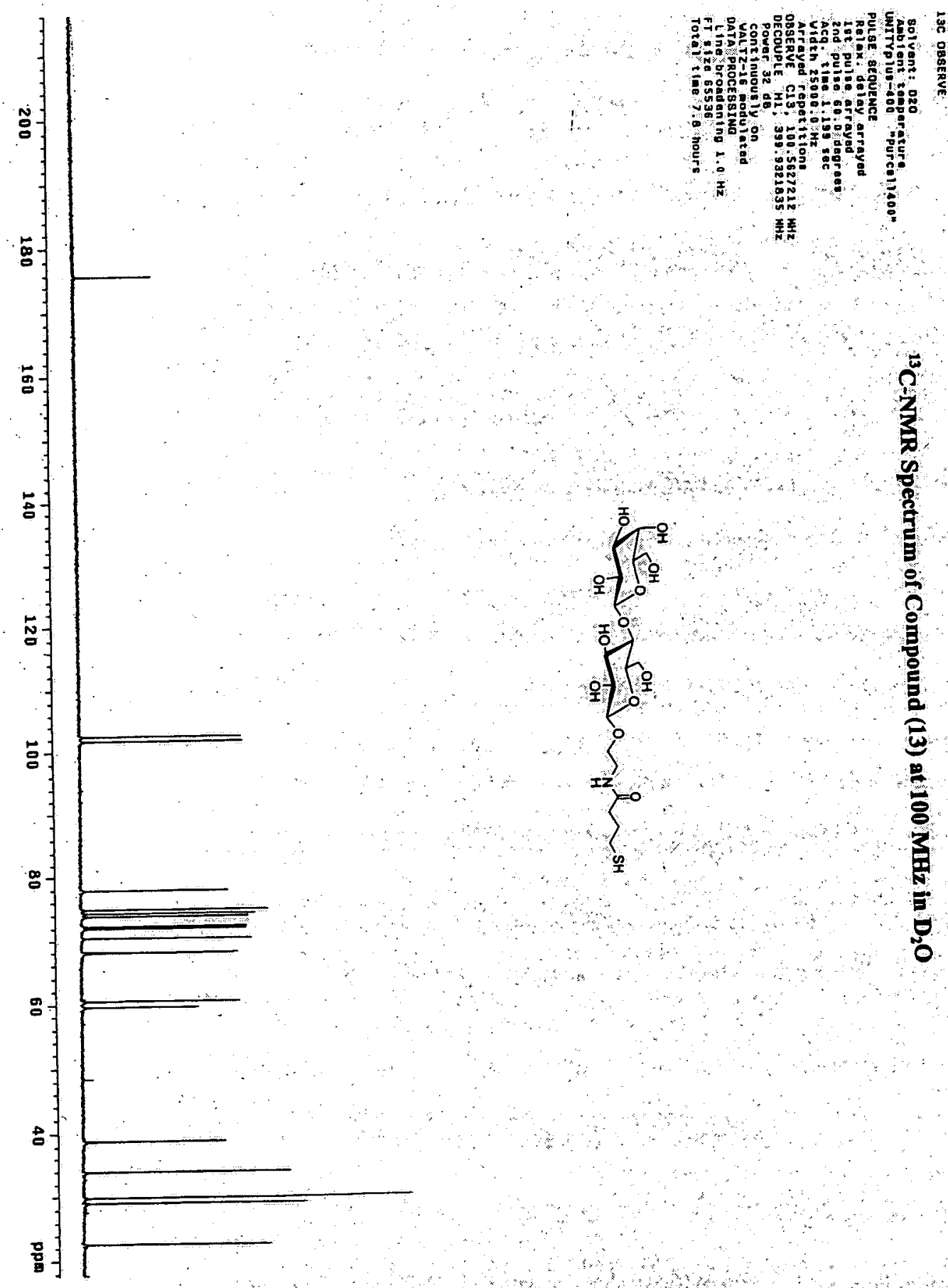
(1) 2004 American Chemical Society, Org. Lett., Gao ol040043a Supporting Info Page 19

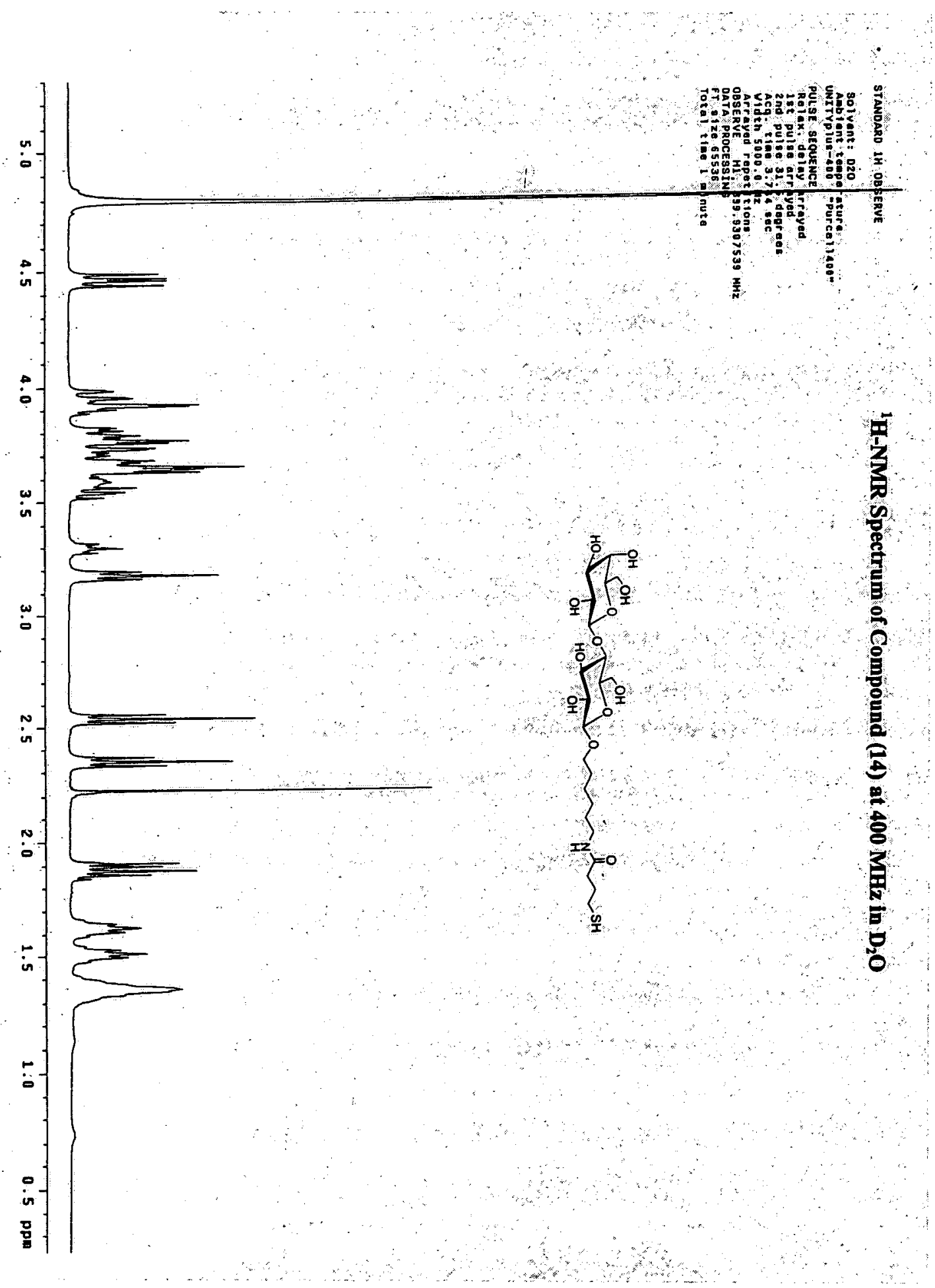


(C) 2004 American Chemical Society, Org. Lett., Gao ol040043a Supporting Info Page 20
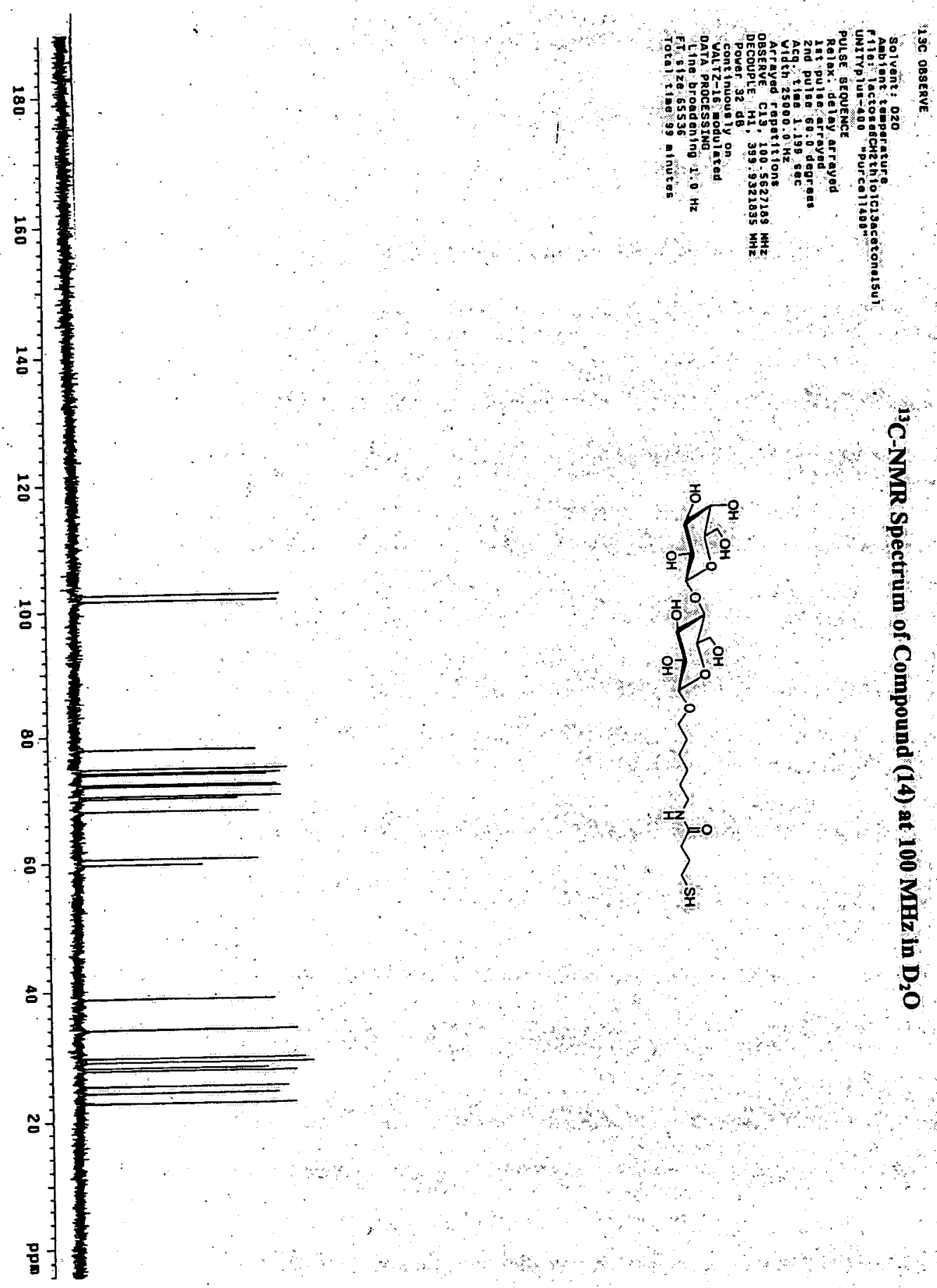
(C) 2004 American Chemical Society, Org. Lett., Gao ol040043a Supporting Info Page 21

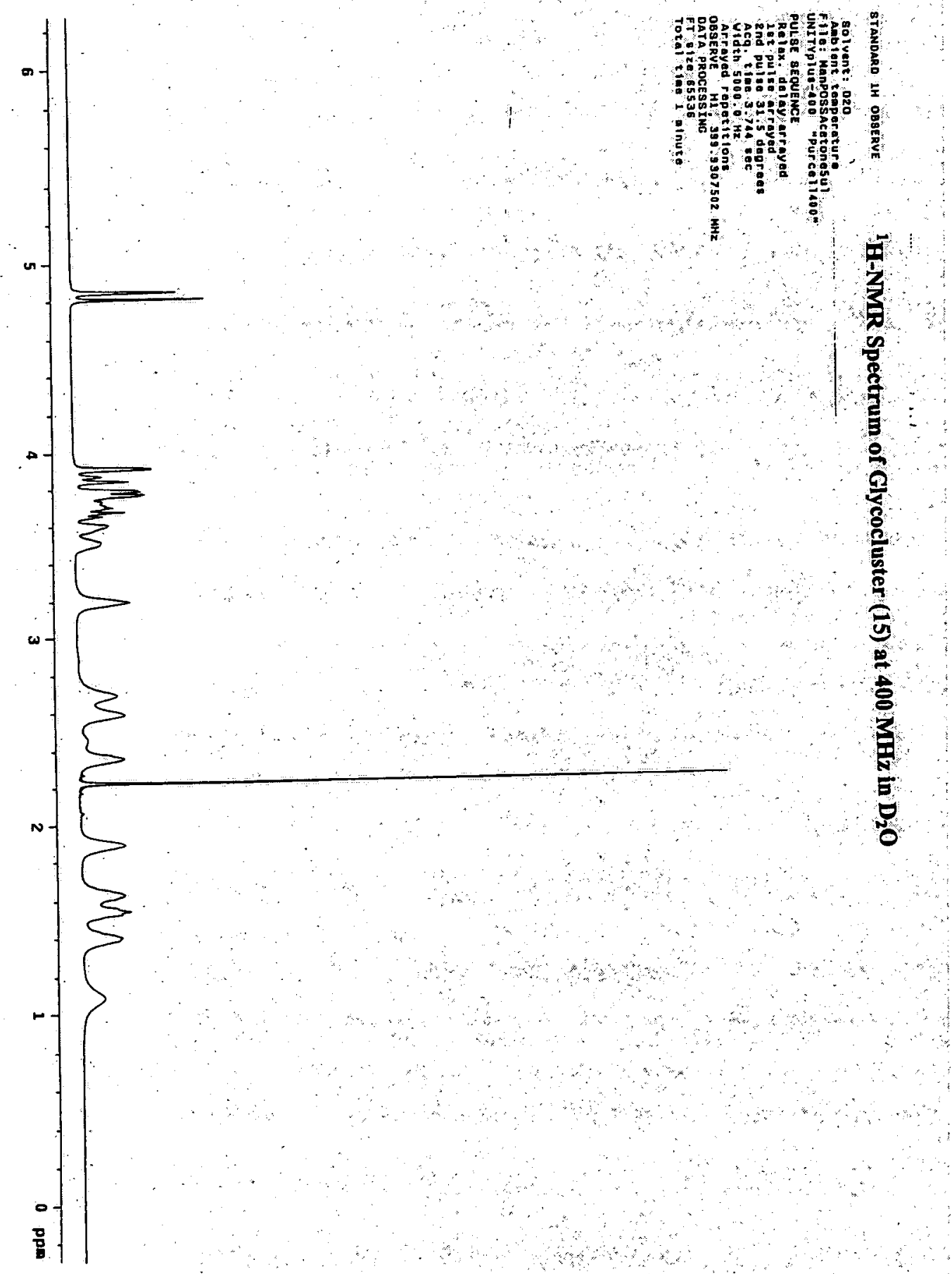


(C) 2004 American Chemical Society, Org. Lett., Gao ol040043a Supporting Info Page 22

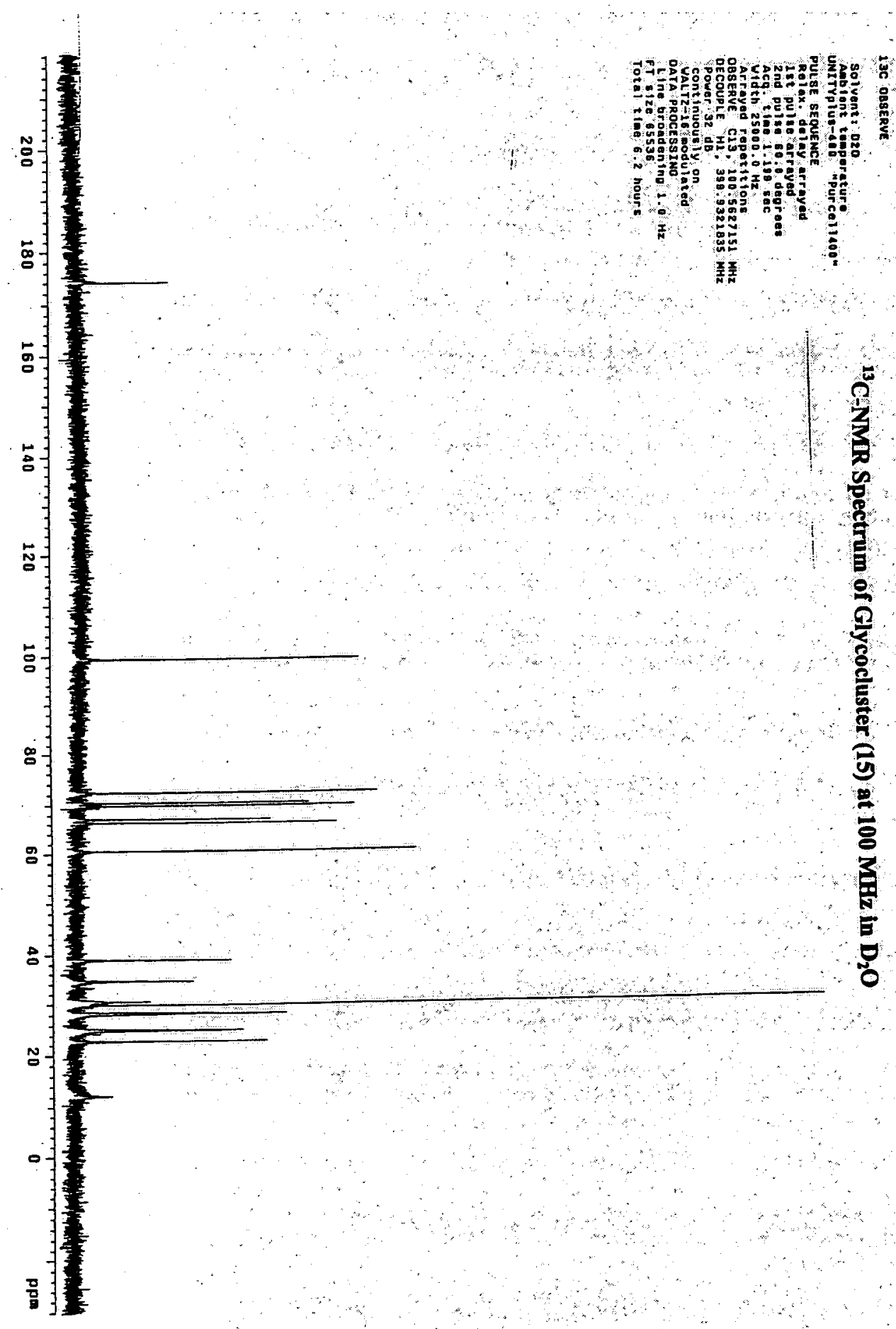


(1) 2004 American Chemical Society, Org. Lett., Gao ol040043a Supporting Info Page 23

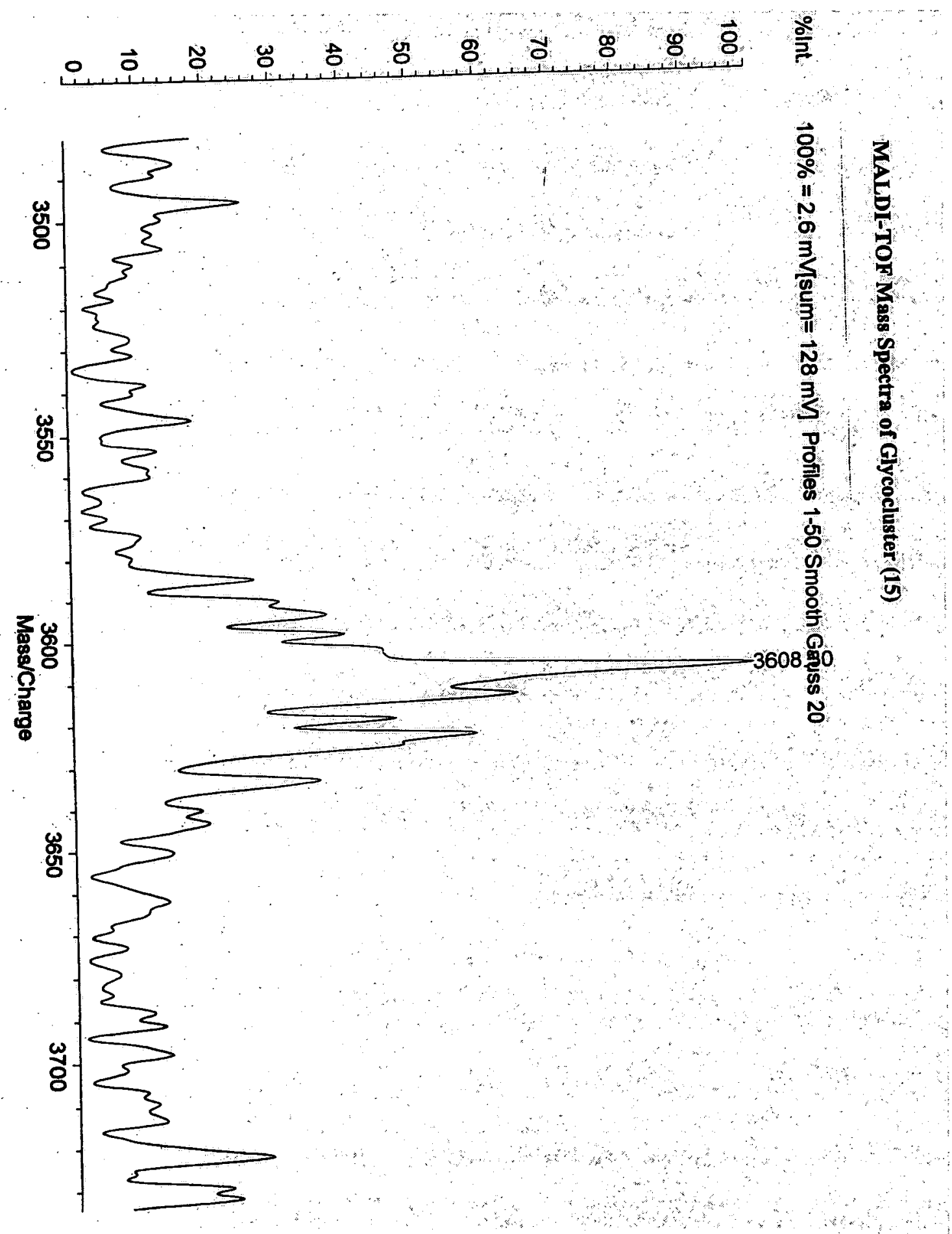


(C) 2004 American Chemical Society, Org. Lett., Gao ol040043a Supporting Info Page 24

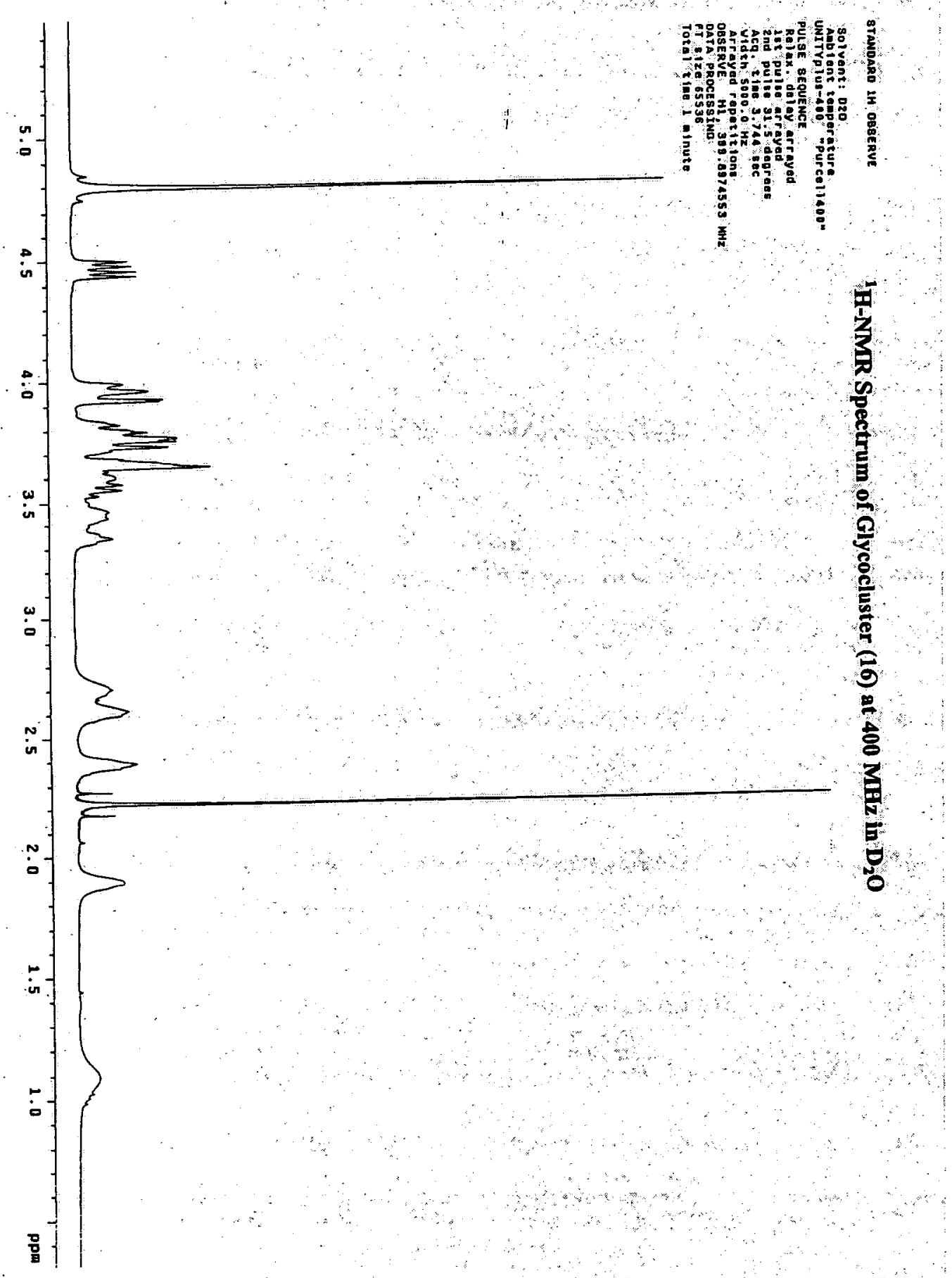


@ 2004 American Chemical Society, Org. Lett., Gao ol040043a Supporting Info Page 25

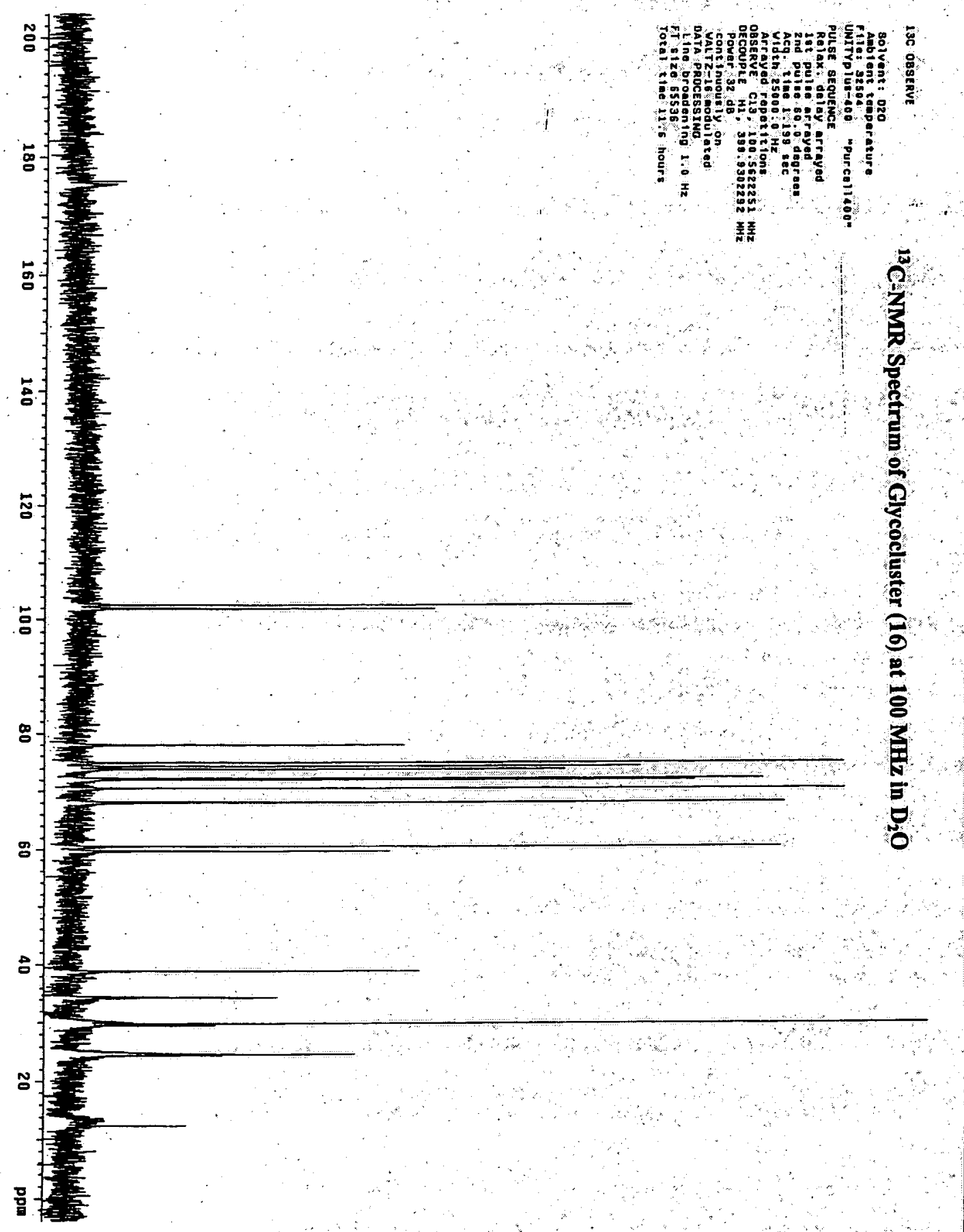


(C) 2004 American Chemical Society, Org. Lett., Gao ol040043a Supporting Info Page 26

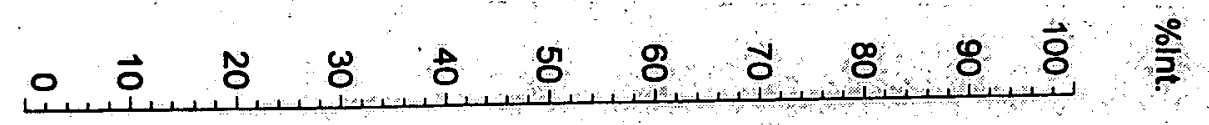

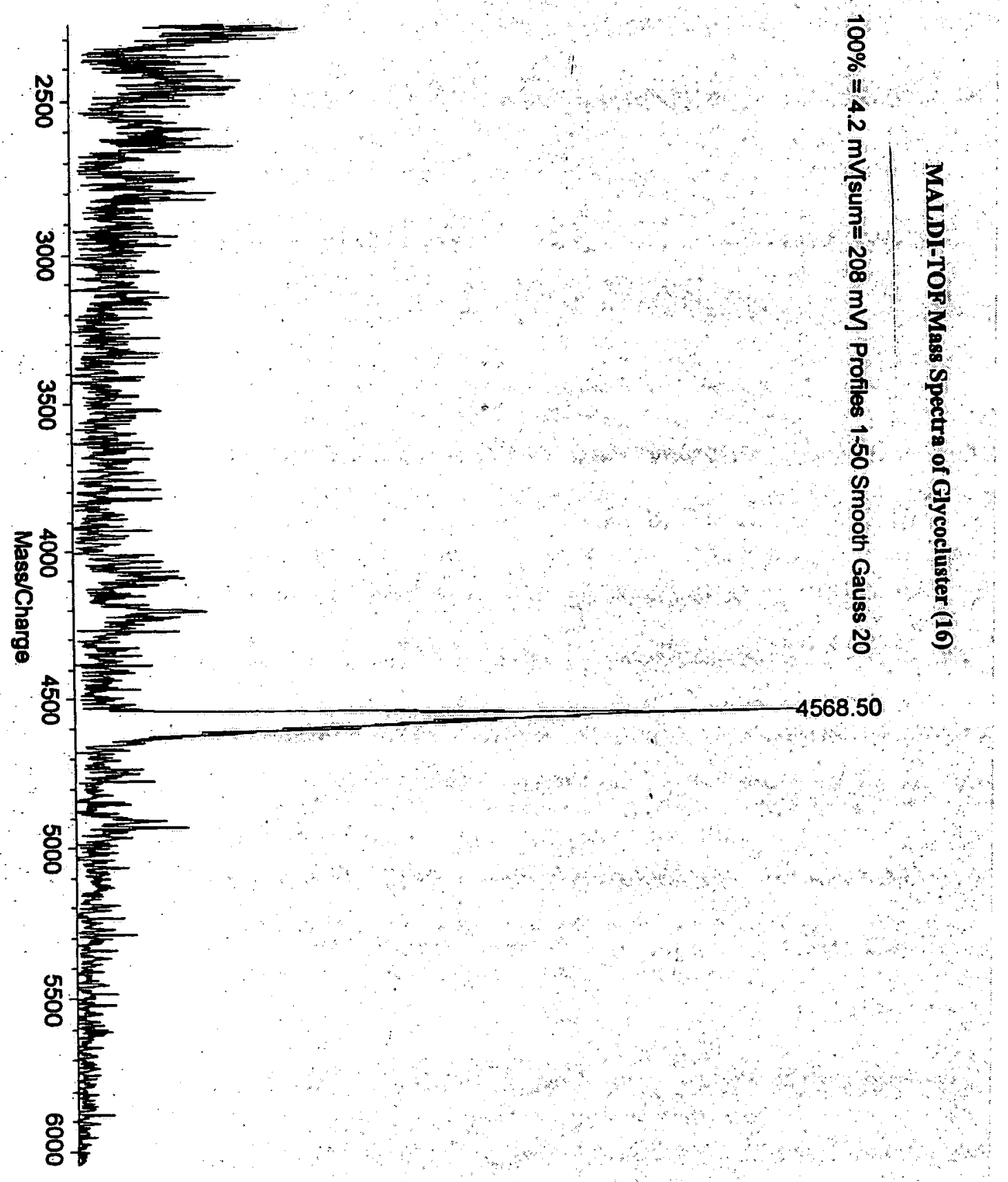


(c) 2004 American Chemical Society, Org. Lett., Gao ol040043a Supporting Info Page 27

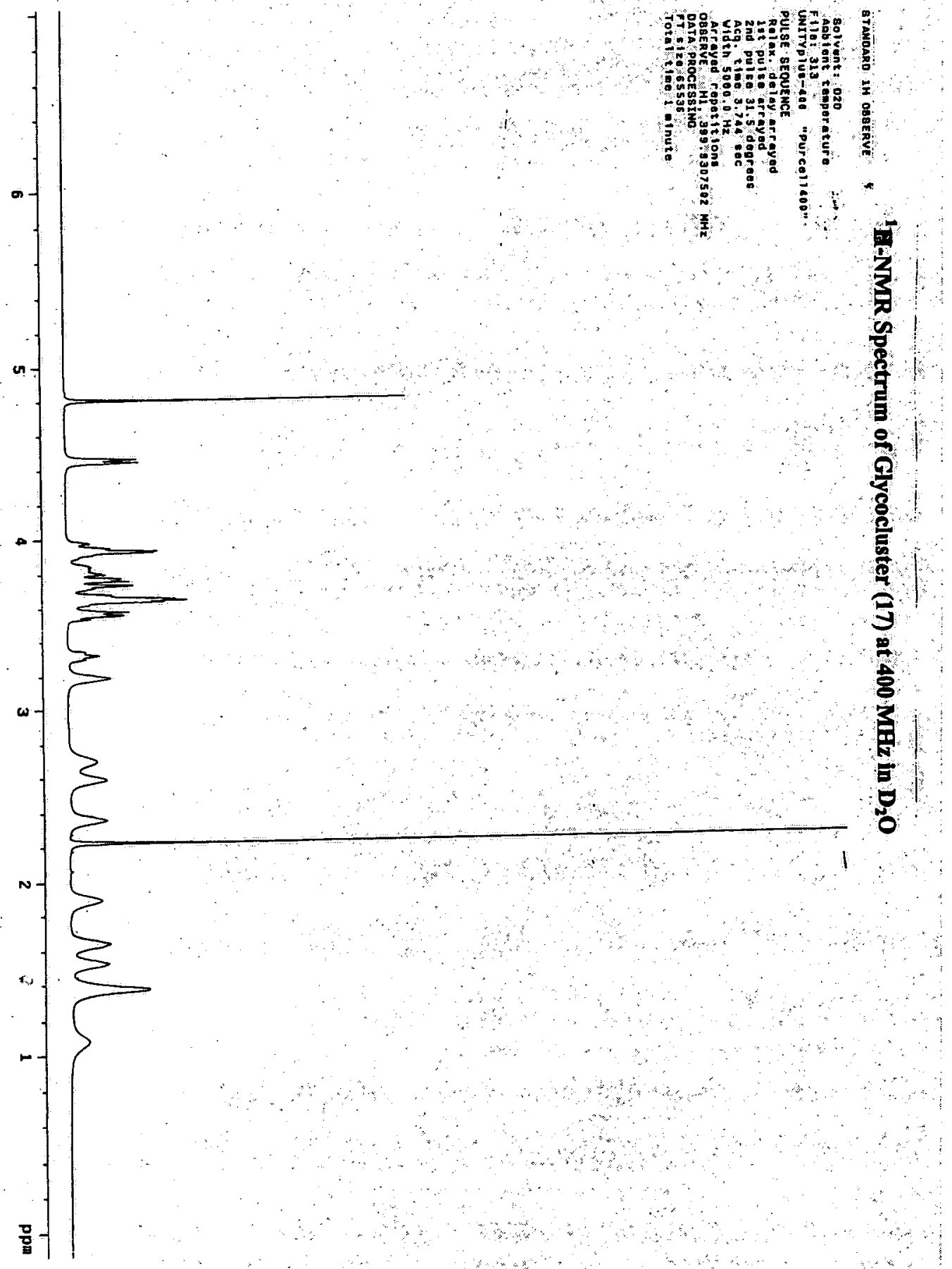


@ 2004 American Chemical Society, Org. Lett., Gao ol040043a Supporting Info Page 28

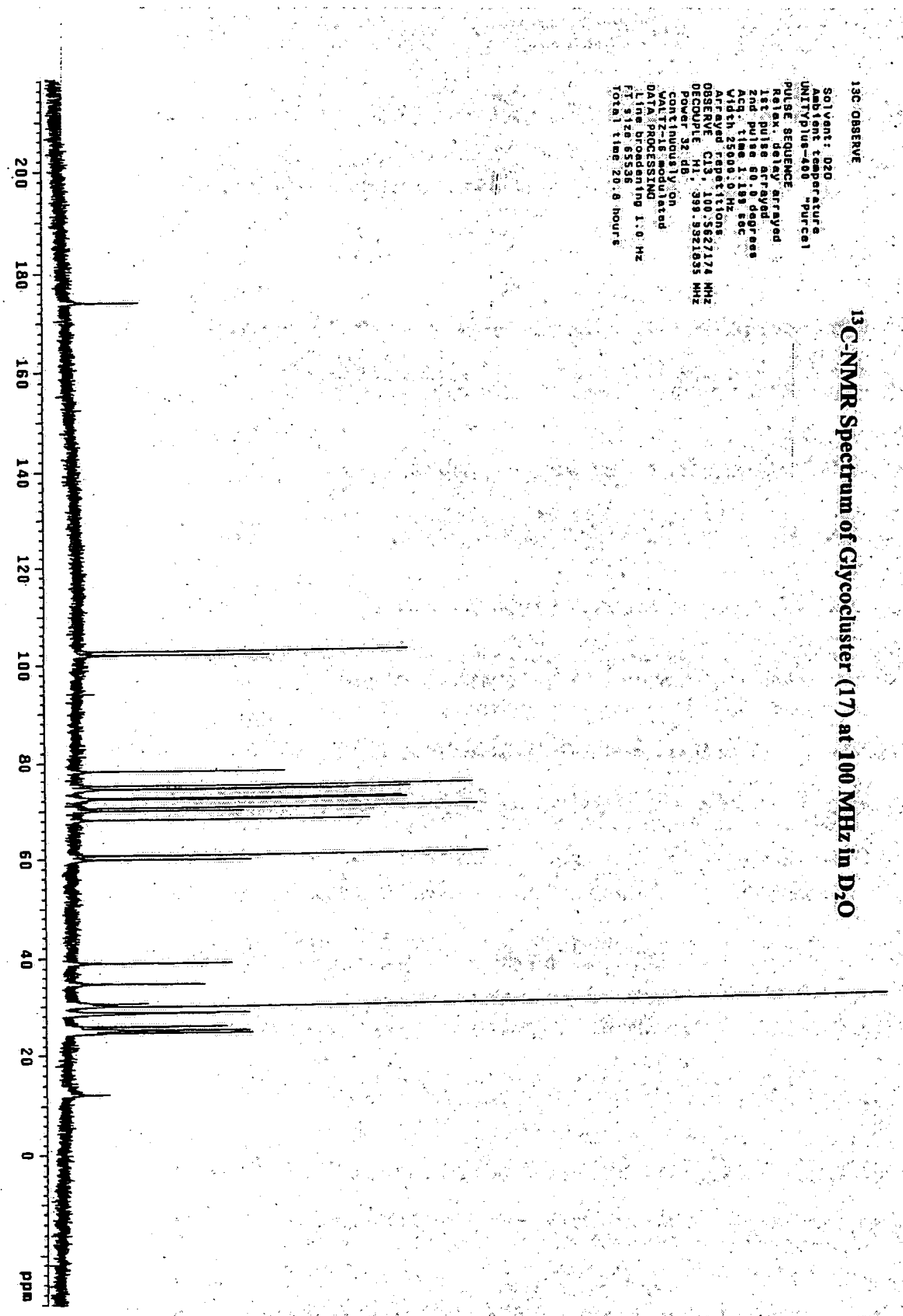


(1) 2004 American Chemical Society, Org. Lett., Gao ol040043a Supporting Info Page 29

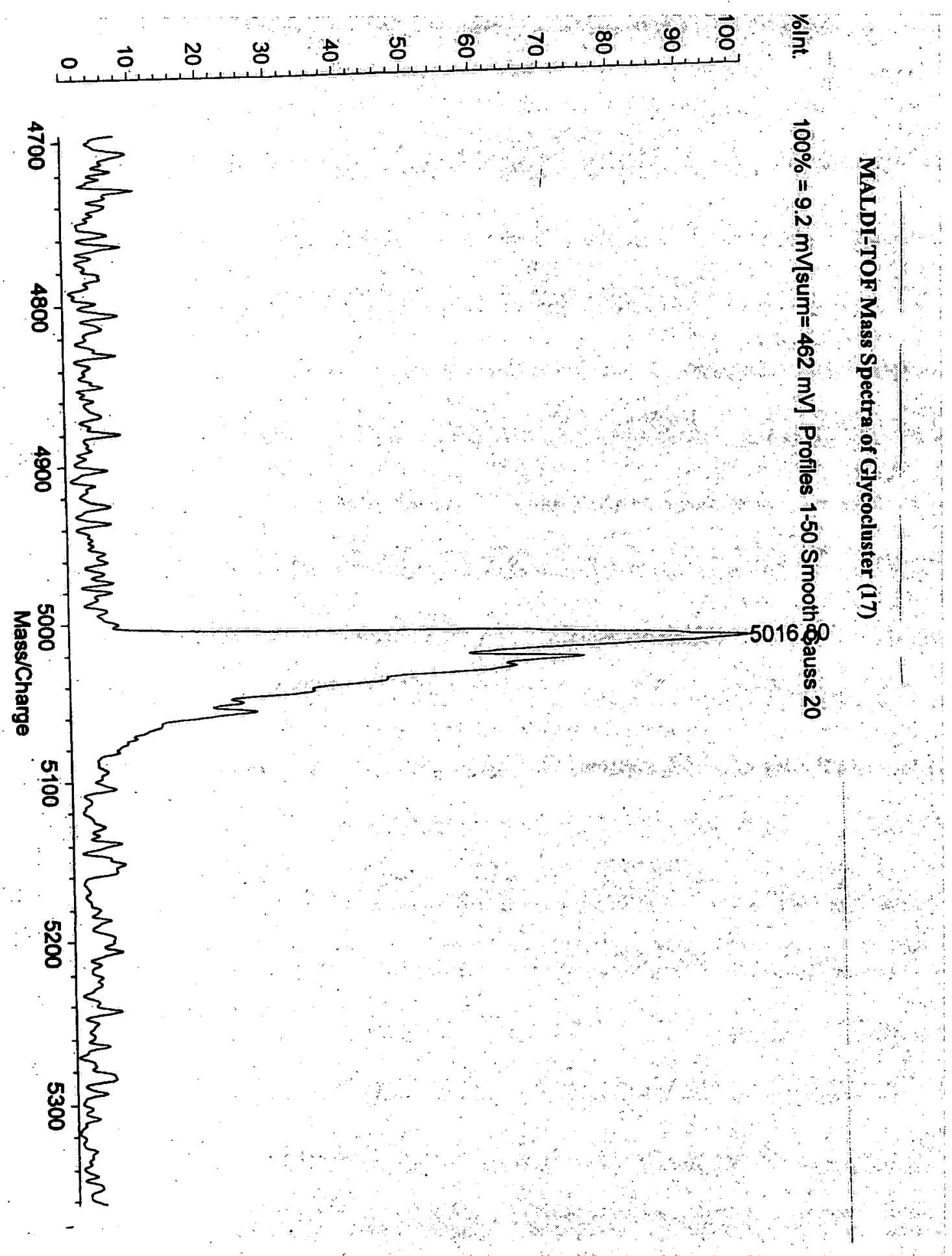

